\title{
Representing the dynamics of a static form
}

\author{
JENNIFER J. FREYD \\ Stanford University, Stanford, California
}

\begin{abstract}
Traditional "feature analysis" theories of letter perception do a poor job of accounting for our ability to read handwritten letters. In this paper, an alternative theory is considered: Perhaps handwriting recognition makes use of information about how letters are formed, as well as of knowledge of static characteristics of letters, such as "distinctive features." In an experiment testing this hypothesis, subjects saw artificial characters drawn in real time and were later asked to identify distorted versions of the same characters presented statically. Subjects were faster on static characters distorted in a manner consistent with the drawing method they had witnessed than on static characters equally distorted but inconsistent with the drawing method. This finding suggests that humans can use dynamic information in the perception of static forms.
\end{abstract}

Letter perception has been investigated extensively by psychologists (for a review, see Gibson \& Levin, 1975). In most cases, the stimuli used, for both experimentation and observation, have been typewritten or machine-produced letters. This choice of stimuli has undoubtedly favored the development of "feature analysis" theories of letter perception, which do not adequately explain how it is that we can perceive handwritten letters. Feature-analysis theories claim that perceivers recognize a given letter by perceiving its "distinctive features," such as whether the letter is symmetric or not, whether it has a left-diagonal line or not, whether the curves are open or closed, etc. One problem with feature-analysis theories is that, although almost none of these distinctive features are truly preserved in handwritten letters, people are clearly able to recognize handwritten messages. Indeed, it is very hard to make a physical description of a given letter that includes all recognizable handwritten examples or "distortions" of that letter and excludes all recognizable handwritten examples of other letters.

There have been some studies that have looked at letter perception in terms of recognizable distortions. One developmental study (Gibson, Gibson, Pick, \& Osser, 1962) used both artificial characters based on printed uppercase letters and transformations (rotations or reversals) of those letters. Gibson et al. found that visual discrimination of letter-like forms improves with

For their encouragement and advice, I warmly thank Jon Baron, Ron Finke, Kathy Hirsh-Pasek, J. Q. Johnson, David Rumelhart, Liz Spelke, and Alf Zimmer, and especially wish to acknowledge the support of Roger Shepard during all stages of this project. I also thank Herbert Clark, Steven Keele, Steven Pinker, and an anonymous reviewer for their helpful comments on earlier drafts of this paper. This research was funded by NSF Grant BNS 80-05517, awarded to Roger Shepard. Requests for reprints should be sent to J. Freyd, who is now at the Department of Psychology, Uris Hall, Cornell University, Ithaca, New York 14853. age as children become better able to reject distortions, and they concluded that what children learn are the "features or dimensions of difference which are critical for differentiating letters" (p. 904). Pick (1965) sought to make an explicit comparison between the distinctivefeatures hypothesis that Gibson et al. supported and an alternative hypothesis, that children learn to match sensory data about letters to prototypes of the letters that they have built up. Pick used the same artificial characters and transformations used by Gibson et al. but she varied the procedure. She taught children to discriminate some of the standard letter-like forms from some of their transformations, and then she specifically compared two types of transfer learning. In the distinctive-features condition, children were asked to discriminate standards they had not yet learned from transformations of those letters of the same type used during training. In the prototype condition, the children were asked to discriminate standards they had not yet learned from transformations of those letters of a type different from that used during training. Pick found that children performed better in the former case, and she interpreted this as support of the distinctivefeatures hypothesis.

In both the Gibson et al. (1962) and Pick (1965) studies, children were asked to discriminate standard forms from distorted forms. Yet, during handwriting recognition, perceivers must be tolerant of distortions of the standard. Also, the specific transformations used in the two studies, those of reversal and rotation, are not of the sort normally encountered in recognizable handwriting. Indeed, the transformations were designed to be in accord with the distinctive-features hypothesis: "the transformations were chosen on an intuitive basis with regard to the distinctive features of letters as a set" (Gibson et al., p. 897). Although feature-analysis theories may account for the ability people have to recognize uppercase printed letters, they do not seem to capture all that is necessary for handwriting recognition. 
An alternative theory of letter perception that may account for our ability to recognize handwritten letters (especially when they are distorted by other letters before or after them) is: Handwriting recognition makes use of information about how letters are formed, as well as, perhaps, of knowledge of static characteristics of letters, such as distinctive features. It may be that perceivers can infer the underlying dynamic pattern of motor movements by applying knowledge of drawing method to the static trace of a handwritten letter. For example, one possibility, based on an analogy to the motor theory of speech perception (see Liberman, Cooper, Shankweiler, \& Studdart-Kennedy, 1967), is that the perceiver produces a model of what he or she is perceiving by following veridical production rules.

The proposal that we use knowledge of drawing methods in handwriting recognition is compatible with some ideas that Watt (1980) expressed on the proper characterization of the alphabet. He talked about letters as being "kinesthetic morphemes" that can be broken down into "kinemes" that are chronologically concatenated. Also, a recent study by Zimmer (1982), which extended the theory proposed here to handwriting production, revealed that people are better able to answer questions about the visual characteristics of a given handwritten letter while forming a "dynamic" mental image than while forming a "static" mental image of that letter. (To form a dynamic mental image of a letter, one imagines the letter being drawn.) Some other investigations of handwriting production sug. gest that even young children have tacit knowledge of drawing methods. For example, it has been found that when children copy geometric figures, such as letters, they usually begin drawing at certain predictable points and proceed in certain predictable directions (for a review, see Simner, 1981). It may be that learning how an alphabet is formed is crucial for learning how to read an alphabet: I first became aware of the importance of knowing how letters are formed when I was learning to read and write Japanese characters. I found that, if I wanted my characters to be recognizable by a native Japanese reader, correct stroke order was important. Printed Japanese characters have stylized indications of stroke order (thick and thin ends, etc.), and when handwritten they often also include thin strokes that only indicate "where the pen moved through the air." Upon examining our own alphabet, I found many similar indications of normal stroke order, including connecting lines between proper strokes. Many type fonts also seem to include stylized indications of normal stroke order.

The initial prediction that I tested here was that familiarity with a writing method should reduce the amount of time required for a perceiver to recognize handwritten symbols consistent with that method. For the experiment, an artificial character set of nine symbols was created. Each character had a proper, or nondistorted, form and a set of distorted versions, anal- ogous to handwritten variants of those symbols. The experiment consisted of a training session lasting about $1 \mathrm{~h}$ and a test session lasting about $15 \mathrm{~min}$.

In the training session, the subjects learned to associate a number with each of the nine artificial characters. In order to separate "familiarity with a writing method" from familiarity with distorted variants, the subjects were never shown distorted symbols in the training session, but were exposed to one of two drawing methods. Nondistorted characters were presented to the subjects in a dynamic fashion, that is, were drawn in real time on a computer-controlled graphics display screen. The subjects were divided equally between two conditions that determined the drawing method, $\mathrm{A}$ or $\mathrm{B}$, that they had witnessed in the training session. All subjects were given the same test, in which half of the distorted characters were predictable distortions with respect to drawing method $\mathrm{A}$, but not $\mathrm{B}$, and the other half, vice versa. The test stimuli were thus counterbalanced: A given item was consistently distorted for half of the subjects and inconsistently distorted for the other half. It was expected that the subjects would more readily identify characters that were consistent with the drawing method to which they had been exposed.

\section{METHOD}

\section{Subjects}

Sixteen Stanford undergraduates were paid for their participation in this study.

\section{Apparatus}

A Megatek 5000 graphics display screen controlled by a Data General Nova computer was used to present visual stimuli to the subjects in both the training and the test sessions.

\section{Training Session}

The training session began with a short introduction in which the nine characters were drawn in numeric order, with the corresponding Arabic numeral in the upper right-hand corner. After the introduction, a randomly selected character was drawn on the screen, but with no corresponding digit, and then was replaced by a question mark in the center of the screen. The subjects identified the character by hitting the appropriate digit on a keyboard. A feedback message appeared and indicated whether the subject was correct or not and what the true answer was. The subjects knew that a block of trials consisted of the nine characters presented in a random order each time, and that the computer would end the training session when they had correctly identified characters from five consecutive blocks. (It took all subjects between 7 and 10 blocks to reach criterion.)

Each character was formed of two or three unconnected linc segments. An average character took about $15 \mathrm{sec}$ to be drawn and remained on the screen an additional $2.5 \mathrm{sec}$ in its final form. In an attempt to imitate human writing, small lags were allowed between the ending of one stroke and the beginning of the next stroke. (There were no apparent motion effects in the display.) The final form of the character on the screen was the same for both drawing methods $\mathrm{A}$ and $\mathrm{B}$. The only difference was the order and/or direction of the strokes used to draw each character. For all characters, drawing method A strokes always were ordered from left to right and were drawn downward. Method B strokes were also mostly ordered from left to right; however, the second or third stroke was drawn upward. See Figure 1 for an example character and the drawing methods. 
DRAWING METHODS

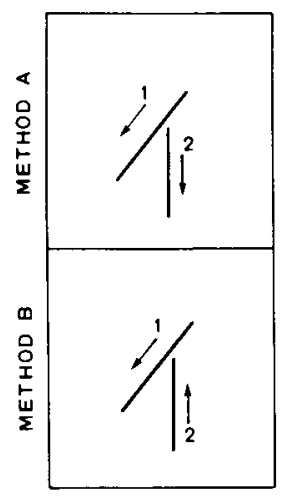

NON-DISTORTED CHARACTER
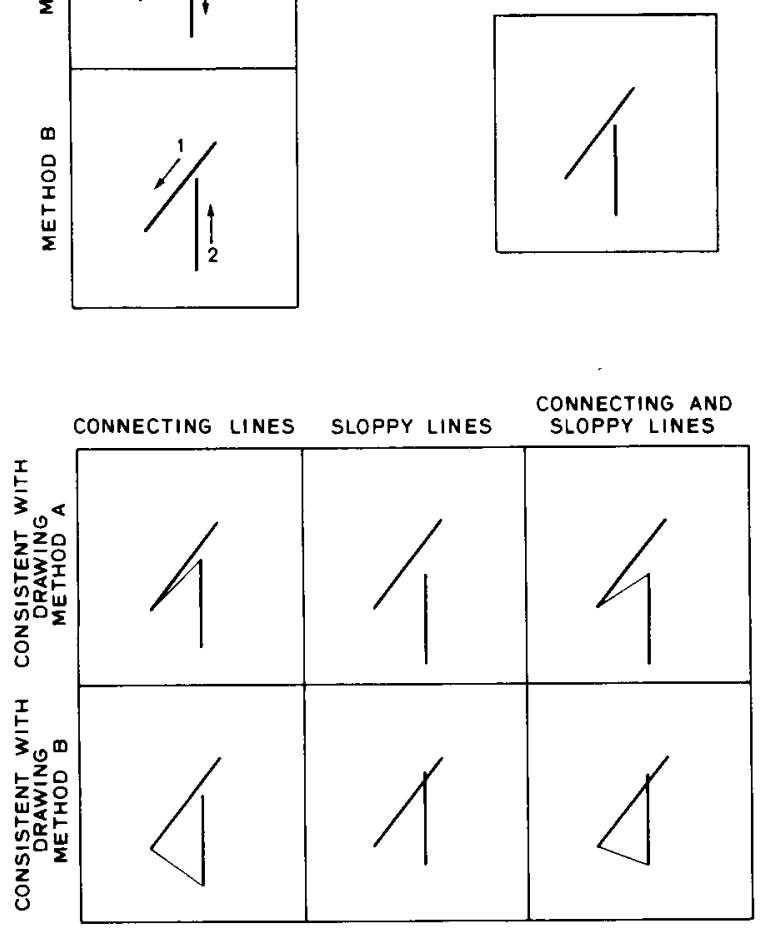

Figure 1. The two drawing methods, the nondistorted test character, and the six distortions also used in the test for an example character. The stroke order ("1" for first stroke) and the stroke direction (parallel arrow) are indicated for the example character.

\section{Test Session}

In the test session, the subjects identified the characters, now presented only in their complete forms, as quickly as possible. It was explained that some of the characters might be slightly distorted, but that the distortions should not interfere with the correct identification of the characters. One of two Arabic digits, which appeared in the upper right-hand corner of each test trial, always corresponded to the character in the center of the screen, but its placement (left or right) was randomly decided for each trial. The subjects used a foot pedal to initiate each trial and a two-button response board to indicate their selection of the right or left digit by pushing the right or left key, respectively.

In preparation of the test stimuli, a set of six distorted characters was generated for each of the nine nondistorted characters, three consistent with each drawing method. As Figure 1 shows, the three distorted characters consistent with any one drawing method were formed such that one had "connecting lines," another had "sloppy lines," and one had both "connecting lines" and "sloppy lines." These distortions were created on the basis of the results of a pilot study in which a different group of subjects were asked to actually draw the characters by using first one and then the other of the drawing methods. The "connecting lines" distortion was based on the finding that writers tended to leave light lines that extended between the end of one proper stroke and the beginning of another. In the "sloppy lines" distortion, the distance between the end point of one stroke and the beginning point of a second stroke was minimized.
Presumably, because of manual laziness, people are likely to make this sort of distortion when actually drawing. The "sloppy lines" distortions also represented the tendency of writers to lengthen the end of a final stroke. Both the minimization of the distance between the end point of one stroke and the beginning point of a second and the extension of the ending position of the second stroke was achieved in the present experiment by simply sliding the position of the "sloppy lines" distorted stroke in one direction or the other, along its own axis. The test session was run in three blocks, the first two of which were practice. (Pilot data revealed that the subjects required at least two blocks of practice before their data settled down; that is, before subjects are familiar with the task, there is a great deal of variability in reaction time from one trial to the next.) Each block had 72 randomly ordered trials: two trials for each of the nine nondistorted characters, and six possible distortions for each of the nine characters.

\section{RESULTS}

The overall error rate was $9.5 \%$. There were no statistically significant differences in error rates between conditions. Only correct reaction times entered subsequent data analyses. Three mean reaction times were calculated for each subject and then were entered into a two-way analysis of variance in which drawing method (A or B) was a between-subjects factor and consistency of distortion (three levels-nondistorted, consistently distorted, inconsistently distorted) was a within-subjects factor. As expected, there was no main effect for drawing method $(F<1)$ and no interaction effect between drawing method and consistency of distortion $(F<1)$. Because drawing method was not originally considered to be a factor (there were two drawing methods so that test stimuli could be counterbalanced across subjects), and because it proved to be wholly nonsignificant, the data were collapsed across drawing method for all subsequent analyses.

A one-way analysis of variance revealed that there were differences among consistently distorted, inconsistently distorted, and nondistorted characters in the predicted way; the subjects took longer to identify inconsistently distorted characters $(1,708 \mathrm{msec})$ than to identify either consistently distorted characters $(1,548 \mathrm{msec})$ or nondistorted characters $(1,547 \mathrm{msec})$. The main effect for distortion (with three levels) was significant $[F(2,30)=3.39, p<.05]$. More importantly, individual comparisons revealed that the $160-\mathrm{msec}$ difference between the reaction times for the inconsistent and the consistent distortions was statistically significant $[F(1,30)=5.09, p<.05]$, as was the 161 -msec difference between the inconsistently distorted and the nondistorted characters $[F(1,30)=5.16, p<.05]$.

As Figure 2 illustrates, the difference between consistent and inconsistent distortions is quite robust; it can be found for all three distortion types. A separate analysis of variance that used only the data for distorted characters was performed. This allowed for a two-way design in which one factor was consistency of distortion (consistent vs. inconsistent) and the other factor was type of distortion ("connected lines" vs. "sloppy lines" vs. "connected lines" and "sloppy lines"). The main 


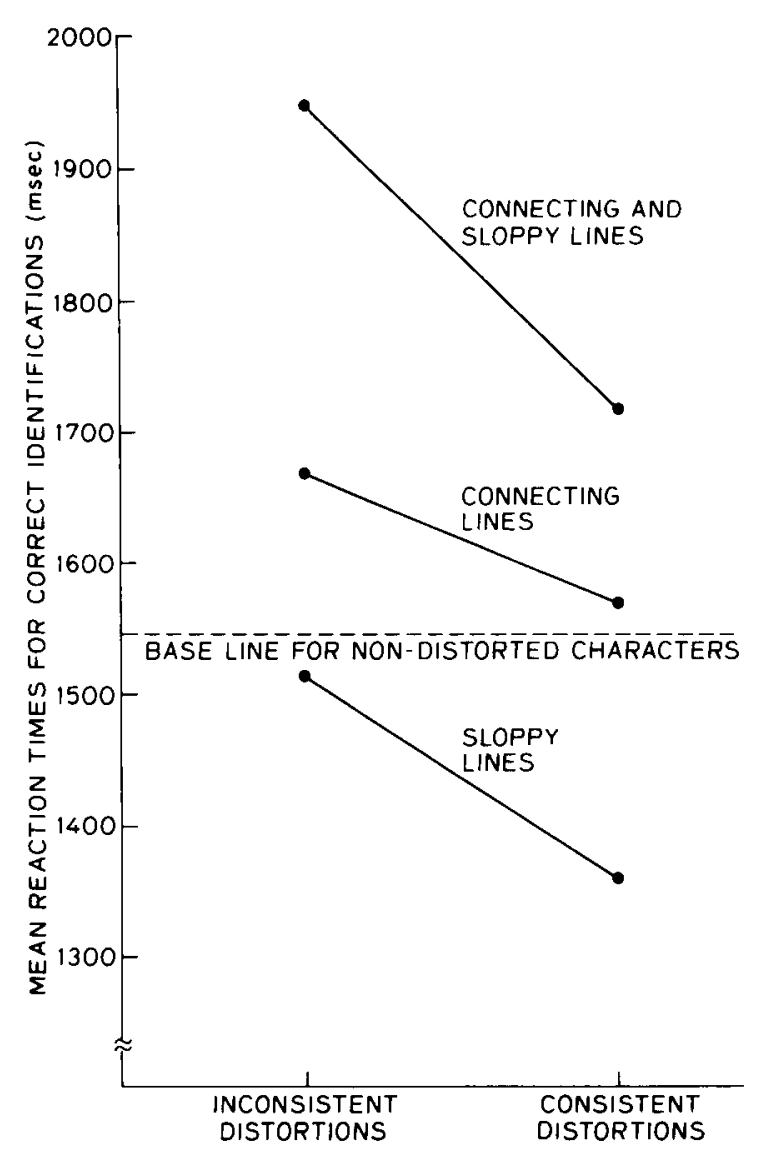

Figure 2 . The results displayed by distortion types.

effect for consistency of distortion was, as predicted, significant $[F(1,15)=5.28, p<.05]$, as was the main effect for type of distortion $[\mathrm{F}(2,30)=12.83, \mathrm{p}<.001]$. Most importantly, the interaction between consistency of distortion and type of distortion was not significant $(F<1)$, which indicated that the difference between consistent and inconsistent distortions could be found for all three distortion types.

Figure 2 also reveals that characters with "sloppy lines" distortions are no harder to identify than the nondistorted characters. Indeed, the inconsistent "sloppy lines" distortions produced almost the same results as the nondistorted characters, which suggests that the "sloppy lines" method of distortion does not grossly distort the configural properties of the character. Yet, the "sloppy lines" distortions do affect identification when they are consistently distorted; this significant facilitation effect in the case of consistent "sloppy lines" distortions compared with nondistorted characters $[t(15)=2.49, p<.05]$ is especially encouraging. It indicates that some kinds of consistently distorted characters are easier to identify than are nondistorted characters, presumably because there is information value in the distortion. That is, if subjects have abstracted the drawing rules for individual characters, one might expect them to find distorted characters that were exaggerated exemplars created by the same rules even easier to identify (see Hyman \& Frost, 1975, for evidence of such a "rule model" in category discrimination).

\section{DISCUSSION}

The finding that knowledge of drawing method influences the recognizability of distorted characters supports the hypothesis that processes of handwriting recognition use tacit knowledge of writing method. Moreover, the results suggest an interesting possibility: Perhaps in general people use knowledge of dynamic processes in the perception of static forms. This conclusion contrasts with most theories of static-form perception in general, and of letter perception in particular. However, it is similar in spirit to some recent theories that perception of objects depends upon a knowledge of the objects' possible transformations (see Shepard, 1981) and is consistent with recent demonstrations that one of the important ways infants determine what makes a given object distinct and unified is to determine what moves as a single object (Spelke, 1982). Indeed, many have argued that the human perceptual system is well adapted for perceiving motion or changes when it is presented with dynamic stimuli (see Gibson, 1966, and Johansson, 1975). The research presented here suggests that in fact we may be so prone to perceive dynamic information that we do so when we are presented with only static stimuli.

An alternative interpretation of the present results is that the drawing method subjects witnessed influenced only the initial encoding of the configural properties of the characters. This explanation would assume that the information stored about the static characters differed between conditions. Although this hypothesis requires post hoc explanations of how initial encoding would be more like the specific "consistent" distortions used in the experiment, it suggests future research that would attempt to distinguish it from the hypothesis that knowledge of drawing method influences perception. Whether the effect of drawing method occurs during initial encoding or during perception, the effect indicates that distinctive-features theories do not account for all of our ability to recognize handwritten letters.

At present, 1 prefer the hypothesis that processes of recognizing static handwritten letters may use tacit knowledge of the manner in which those letters are drawn and that in general humans perceiving static forms may be using tacit knowledge of dynamic aspects of those forms. This general hypothesis can be tested only by looking at perceptual situations other than letter perception. Recently, I have been studying the perception of photographs: I have found that subjects apparently represent the dynamics of static photographs that depict objects in the course of some motion (Freyd, 1983). When subjects are asked to hold such a picture in memory, they seem to anticipate the motion in the 
scene, for they find it harder to correctly reject distractors as "different" when the distractors are photographs of the same scene but shot later in time than when the distractors are photographs of the scene but shot earlier in time.

In conclusion, knowledge of drawing method can predictably influence the recognizability of distorted characters. This suggests that the importance of representing motion or change goes beyond the cases in which dynamic stimuli are present. Future research that explores these hypotheses further is indicated, both within the domain of handwriting recognition and in other areas of mental representation.

\section{REFERENCES}

FREYD, J. J. The mental representation of movement when viewing static stimuli. Perception \& Psychophysics, 1983, 33, 575-581.

Gibson, E. J., Gibson, J. J., Pick, A. D., \& Osser, H. A developmental study of the discrimination of letter-like forms. Journal of Comparative and Physiological Psychology, 1962, 55, 897-906.

Gibson, E. J., \& Levin, H. The psychology of reading. Cambridge, Mass: MIT Press, 1975.
Gibson, J. J. The problem of temporal order in stimulation and perception. Journal of Psychology, 1966, 62, 141-149.

HYMan, R., \& Frost, N. Gradients and schema in pattern recognition. In P. M. A. Rabbitt \& S. Dornic (Ed.), Attention and performance $V$. New York: Academic Press, 1975.

Johansson, G. Visual motion perception. Scientific American, 1975, 232(6), 76-88.

Liberman, A. M., Cooper, F. S., Shankweileh, D. S., \& Studdant-Kennedy, M. Perception of the speech code. Psychological Review, 1967, 74, 431-461.

Pick, A. D. Improvement of visual and tactual form discrimination. Journal of Experimental Psychology, 1965, 69, 331-339.

ShePARD, R. N. Psychophysical complementarity. In M. Kubovy \& J. R. Pomerantz (Eds.), Perceptual organization. Hillsdale, N.J: Erlbaum, 1981

Simner, M. L. The grammar of action and children's printing. Developmental Psychology, 1981, 17, 866-871.

SPElke, E. S. Perceptual knowledge of objects in infancy. In J. Mehler, M. Garrett, \& E. Walker (Eds.), Perspectives in mental representation. Hillsdale, N.J: Erlbaum, 1982.

WATT, W. C. What is the proper characterization of the alphabet? II: Composition. Ars Semeiotica, 1980, 3, 3-46.

Zimmer, A. Do we see what makes our script characteristic or do we only feel it? Modes of sensory control in handwriting. Psychological Research, 1982, 44, 165-174.

(Manuscript received December 6, 1982; revision accepted for publication March 11, 1983.) 